\title{
Recurrent Abdominal Wall Inflammation After Resection of Urachal Xanthogranuloma
}

\author{
Sung Yong Cho
}

Department of Urology, Inje University Ilsan Paik Hospital, Inje University College of Medicine, Goyang, Korea

\begin{abstract}
We report a very rare case of urachal xanthogranuloma. In this case, the inflammation recurred after 9 months even though it was removed by surgical resection. A 43-year-old woman presented with low abdominal walnut sized round mass, mild abdominal pain, and no specific voiding symptoms. Through radiologic evaluation with computed tomography (CT), we observed an infiltration in surrounding tissues of urachal mass from anteriosuperior aspect of bladder until umbilicus, so urachus inflammation as well as malignant tumor of urachus origin was suspected. Urachal mass excision and partial cystectomy were conducted with low midline incision and it was reported pathologically as xanthogranulomatous inflammation. Afterwards the patient lived without inconvenience but after 9 months she returned because of pain and palpable nodule in the right lower abdominal area. Despite taking antibiotics, there was no improvement and abscess formation was observed in CT. Finally, its symptoms improved after abscess incision and drainage. (Korean J Urol Oncol 2018;16:82-85)
\end{abstract}

Key Words: Urinary bladder • Urachus · Xanthogranuloma

Urachal xanthogranulomatous inflammation is a very rare disease. Among the papers published up to now, 2 cases were reported in Spain, 13 cases in Japan, ${ }^{3-5} 1$ case in Taiwan ${ }^{6}$ and 2 cases in $\mathrm{Korea}^{7,8}$ respectively and we report the third case of Korea with this paper. In the case of this patient, a serious abscess formation recurred unusually in the same area after 9 months.

\section{CASE REPORT}

A 43-year-old woman visited hospital because of a palpable mass about the size of wallnut as chief complaint. The patient

Received May 16, 2018, Revised May 24, 2018,

Accepted May 27, 2018

Corresponding Author: Sung Yong Cho

Department of Urology, Inje University Ilsan Paik Hospital, Inje University College of Medicine, 170 Juhwa-ro, Ilsanseo-gu, Goyang 10380, Korea

E-mail: csy1204@paik.ac.kr

Tel: +82-2-910-7230, Fax: +82-2-910-7118

ORCID code: https://orcid.org/0000-0002-4870-829X had a laparoscopic appendectomy 3 months ago because of appendicitis and after 2 weeks a thumb-size mass was found at lower abdominal area for the first time. In the then evaluation with ultrasonography, cystic mass escaped from bladder until abdominal wall was observed and the patient was diagnosed for the first time with cystic mass due to urachus patency at that time. She had no previous history of diabetes and hypertension, and her body mass index was $22.3 \mathrm{~kg} / \mathrm{m}^{2}$ in normal range. Afterwards, even she was treated with antibiotics because the mass has been increased, there was no improvement and she was transferred to our hospital. At the time of her visit, a lower abdominal mass about the size of walnut was felt in the middle of the lower abdominal area with slight redness and mild tenderness but there was no special voiding symptom. In enhanced abdominal pelvic CT, Ill-defined enhancing lesion $(2.2 \mathrm{~cm} \times 1.8$ $\mathrm{cm})$ with perilesional infiltration and adjacent peritoneum thickening in pelvic peritoneum, anterosuperior aspect of urinary bladder were observed and this mass combined with diffuse enhancement of rectus abdominis muscle fascia and extends to

(c) (i) (-) This is an Open Access article distributed under the terms of the Creative Commons Attribution Non-Commercial License (http://creativecommons.org/licenses/by-nc/4.0/) which permits unrestricted non-commercial use, distribution, and reproduction in any medium, provided the original work is properly cited. 2018 (C) Copyright The Korean Urological Oncology Society and The Korean Prostate Society. All Rights Reserved. 
properitoneal fat, rectus abdominis muscle, and subcutaneous layer of low abdomen (Fig. 1).

Therefore, we could not exclude urachal origin inflammatory change such as complicated cyst, granulomatous infection such as actinomycosis or tuberculosis and urachus origin malignant tumor. Also, inflammatory change or metastasis was suspected because several enhancing nodular lesions in right lower quadrant (RLQ) region and pro-peritoneal fat layer (max: $1.6 \mathrm{~cm} \times$ $1.6 \mathrm{~cm}$ ) were observed additionally in RLQ area. According to the blood test when the patient visited hospital, it were reported that complete blood count: white blood cell (WBC), 6,390/ $\mu \mathrm{L}$; C-reactive protein (CRP), $4.0 \mathrm{mg} / \mathrm{L}$; urine analysis: red blood cell may/high power field (HPF), WBC 1-4/HPF, no bacteria/HPF. Because the complaint from the patient was not serious, we administered cephalosporin and quinolone group antibiotics in consecutive order for 2 weeks, however the patient was not improved, and we conducted exploratory laparotomy with low midline incision and we observed a severe adhesion in the abdominal wall. The mass was extended from abdominal wall until anterosuperior wall of bladder and caused adhesion around until posterior rectus wall and peritoneal wall.
Therefore, we should remove until surrounding tissues in order to excise urachal mass, and also, we removed partial bladder, posterior rectus fascia, and a part of peritoneal wall. During that operation, we took a culture specimen from the pus in the mass and it was reported as Bacterioides fragilis. The final diagnosis was cyst with xanthogranulomatous inflammation according to the pathological report (Fig. 2).

The patient was discharged at the 7th hospital day after all symptoms improved. But the patient returned after 9 months because of the pain and the palpable mass about the size of egg in the RLQ area. The redness was spread around the mass with a severe tenderness. In CT evaluation, we found a $3.3 \times 2.7-\mathrm{cm}$-sized irregular enhancing lesion which invaded pro-peritoneal fat layer. Through the quinolone antibiotics treatment for 2 months, the pain was improved and the size of mass was reduced. The CRP checked at that time was $0.6 \mathrm{mg} / \mathrm{L}$. After 4 months of monitoring the patient condition, the pain in the right lower abdominal area occurred and the mass increased in the same area. Accompanied with fever and chill, the CRP of blood test was increased to $8.8 \mathrm{mg} / \mathrm{L}$. And the enhanced lesion observed in the previous CT increased to 5.4
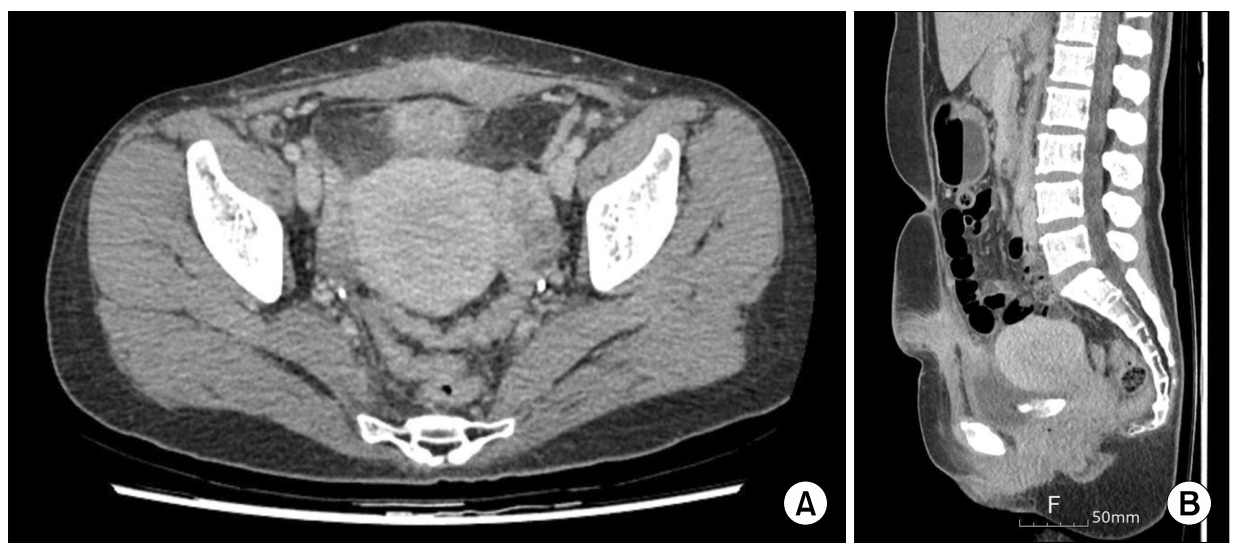

Fig. 1. Abdominal pelvic computed tomography: transverse image (A) and sagittal image (B), showing Ill-defined enhancing lesion $(2.2$ $\mathrm{cm} \times 1.8 \mathrm{~cm})$ with perilesional infiltration anterosuperior aspect of urinary bladder.
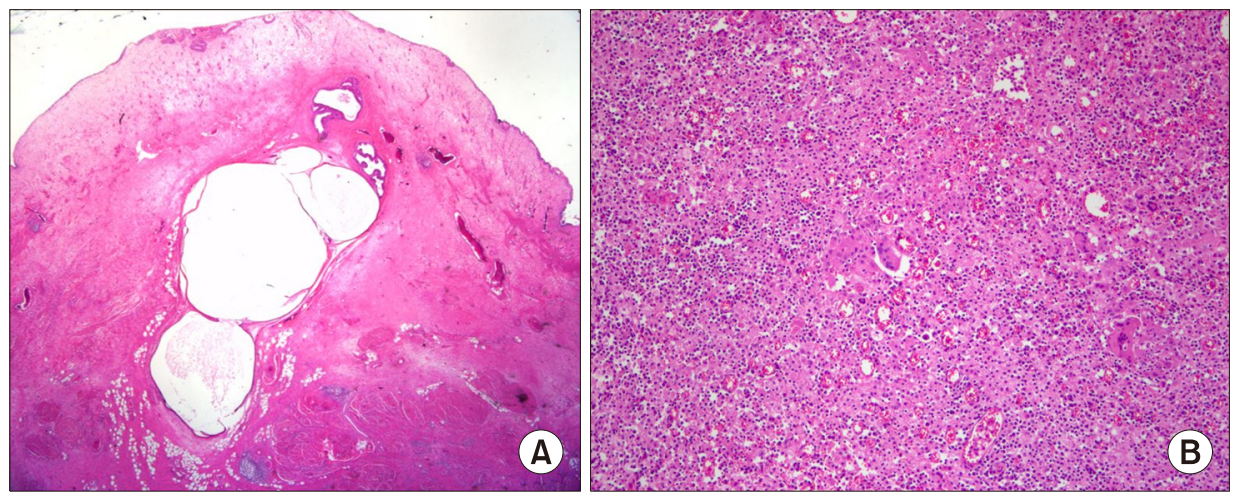

Fig. 2. (A) Urachal cyst shows thick fibrous cyst walls and flat and glandular bland-looking epithelial cells $(H \& E, \times 10)$. (B) Xanthogranulomatous inflammation was noted focally in the cyst wall. Xanthoma cells and foamy histiocytes with lymphoplasma cells are mixed (H\&E, $\times 100)$. 


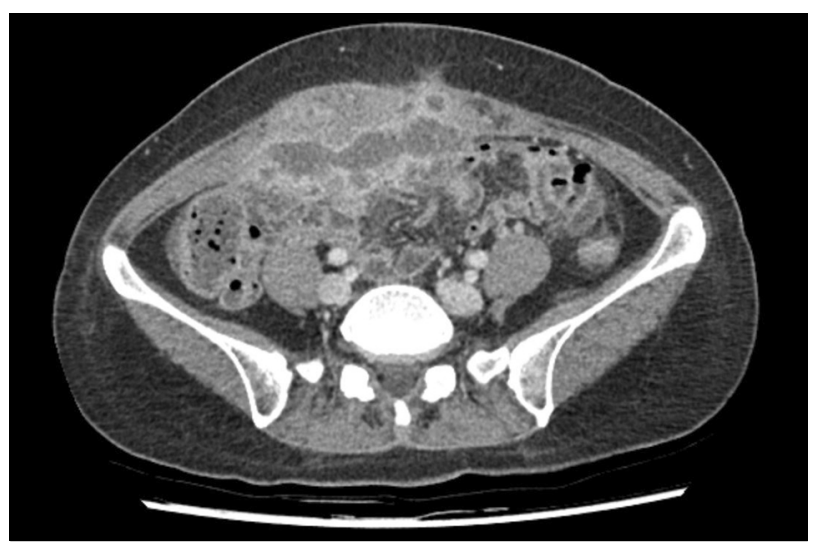

Fig. 3. Progression of irregular enhancing lesions with internal low densities in right lower quadrant area, invading properitoneal fat layer $(3.9 \mathrm{~cm} \times 3.1 \mathrm{~cm} \rightarrow 5.4 \mathrm{~cm} \times 2.9 \mathrm{~cm})$.

$\mathrm{cm} \times 2.9 \mathrm{~cm}$ (Fig. 3).

In case of urine and blood culture, any bacteria were found. Even using antibiotics such as etrapenem and tazobactam, there was no improvement, and we drainaged the abscess by inserting penrose drain into the incised wound. In the pus culture, coagulase negative Staphylococcus was detected. After 7 days when the symptoms were improved, we changed to closed drain and closed the wound. Then after another 7 days, we removed the drain and all symptoms were improved.

\section{DISCUSSION}

Xanthogranulomatous disease is well known as the pathologic finding of large clusters of macrophages with lipid-rich cytoplasm. This kind of lesion appears in the different areas of body. For example, it was reported that this kind of lesion appears well in mandible, retroperitoneum, gallbladder, lung, stomach, pericardium, ovary, etc. and urologically, it was relatively common in pyelonephritis and xanthogranuomatous cystitis also was reported once in a while. ${ }^{9}$ But, the case in urachus is very rare and up to now 13 cases in Japan, ${ }^{3-5} 2$ cases in Spain, ${ }^{1,2} 1$ case in Taiwan $^{6}$ and 2 cases in Korea were reported. ${ }^{7,8}$ In all cases mentioned before, the common point was that all case found a palpable mass in the lower abdominal area, but related to the pain, the cases were mixed having the case with severe, mild and no pain and nearly half of the cases did not show fever, chill, voiding symptoms. Also, there were some cases with previous surgical history, but not others before the mass was found. The age of patients was also varied from
18 to 75 years old and most of the cases not mentioned about underlying disease. It seems that there was no difference in male and female, but the number of cases in not enough to impart a statistical meaning. In our case also the pain and the fever were not severe at the beginning and there were no special voiding symptoms and any pyuria was detected in urine analysis. But we consider that initial symptoms and examination results may be different because in the majority of cases this kind of patient is transferred after treated with antibiotics in primary care center. As pathogenesis of xanthogranulomatous cystitis, there are chronic infection, foreign materials such as suture materials, malignant bladder tumor, immunologic disorders, abdominal lipid metabolism, etc., and because we consider that the pathogenesis of kidney is similar, in case of urachus also will be similar. ${ }^{9,10}$ In this case, the patient had laparoscopic appendectomy several months before this disease and we may suspect that this surgical procedure caused the disease, but it is not clear yet. Enhanced abdominal pelvic CT is helpful for the diagnosis but as all cases we may not exclude the malignant disease of urachus. Therefore, the final diagnosis we defined by pathologic report after resection of urachal mass and partial cystectomy and in this case we did at the same way. In other cases, there was no problem with prognosis after the first resection, but in this case, after a long time of 9 months there was abscess formation and Staphylococcus was detected in abscess pus, however it is difficult to consider that the patient was infected at the first resection because the symptoms appeared after a long interval. This situation makes to consider that the first resection can be applied as pathogenesis, but we cannot conclude it as pathogenesis because we did not make a pathologic confirm.

Because xanthogranuloma may develop recurrent inflammatory disease even after a long period of primary treatment, periodic follow-up is necessary.

\section{CONFLICT OF INTEREST}

The author claims no conflicts of interest.

\section{REFERENCES}

1. Carrere W, Gutiérrez R, Umbert B, Solé M, Menéndez V, Carretero P. Urachal xanthogranulomatous disease. $\mathrm{Br} \mathrm{J}$ Urol 1996;77:612-3. 
2. Díaz Candamio MJ, Pombo F, Arnal F, Busto L. Xanthogranulomatous urachitis: CT findings. J Comput Assist Tomogr 1998;22:93-5.

3. Kasai T, Miyake N, Fukukawa T, Hirakawa E. A case of urachal xanthogranuloma causing recurrent intestinal obstruction. Hinyokika Kiyo 2001;47:587-90.

4. Kinebuchi Y, Nakazawa M, Fujiwara M, Yoneyama T. Urachal xanthogranuloma caused by a swallowed fish bone: a case report. Hinyokika Kiyo 2001;47:797-800.

5. Yamamoto T, Mori Y, Katoh Y, Iguchi M, Minamidani K, Sawai $\mathrm{Y}$, et al. A case of urachal xanthogranuloma suspected to be a urachal tumor. Hinyokika Kiyo 2004;50:493-5.

6. Tseng SF, Yang WC, Sung MT. Urachal xanthogranuloma- tous inflammation. Urol Sci 2010;21:187-8.

7. Park S, Ji YH, Cheon SH, Kim YM, Moon KH. Urachal xanthogranuloma: laparoscopic excision with minimal incision. Korean J Urol 2009;50:714-7.

8. Oh JH, Chung JM, Choi S. Xanthogranulomatous inflammation of urachus. Korean $\mathrm{J}$ Urogenit Tract Infect Inflamm 2013;8:55-8.

9. Walther M, Glenn JF, Vellios F. Xanthogranulomatous cystitis. J Urol 1985;134:745-6.

10. Bates AW, Fegan AW, Baithun SI. Xanthogranulomatous cystitis associated with malignant neoplasms of the bladder. Histopathology 1998;33:212-5. 